the sulphate of baryta. This result we can count upon with infallible certainty. Given as a purgative, we cannot be sure of its action, although its power should be as certain and definite in the human frame as in the test-tube. The reason that we cannot be sure of its action as a remedy is because of differences in the conditions under which it is acting. It is our business to find out these conditions, so that, when we, meet them again, we may know how to meet them. For there is an invariable relation between cause and effect, as invariable as the relation between an unchecked falling stone and the earth. We are, as I have said, just learning in medicine the paramount importance of tracing effects to their true cause. We may laugh at the credulity of the Middle Ages; when the way to cure a sword-wound was to apply a salve to the sword; when the only reason for the pestilence was the eccentric advent of some wandering fiery comet. But it is only with our own day that we improve upon the knowledge of our fathers ; that, disdaining witcheraft, and spells, and delusions, and charms, and fables, we begin to see that the laws under which our bodies live are as immutable as the laws which govern Jupiter and the Pleiades; that, when we break them, we must suffer the penalty; that every infraction has its penalty. We learn that there is no such thing as an effect.without a cause; we learn, too, that the penalty for breaking one law will not attend the violation of another. It would be absurd to say that if a child killed a fly it would have a pain in the stomach, and yet the time has been when such a statement would have been logical according to the accepted logic of medicine. The child may kill a fly-the child may have a pain in the stomach; but the pain does not result from the deed. The deed may lead to the pain in this, that the child may have to go dinnerless as a punishment for cruelty, and may gorge itself with green apples to supply nature's needs. But the penalty would be for eating unripe fruit, not for killing flies. In the same way, an unrighteous community given to cheating, lying, and profane speech may be visited by an epidemic. But the epidemic would come, not as the penalty for broken scriptural commandments, but as the penalty for broken sanitary laws, the penalty for impure water, imperfect drainage, or communication with an in. fected district. The violation of scriptural laws may lead to buildings being scamped, to imperfect drains, and a polluted water-supply. But simple repentance for these sins will not stop the epidemic until the sanitary laws are observed. The laws of nature admit of no exception and no excuse. The rain falls alike on the just and the unjust. If a viaduct break and a train be thrown into the river or the valley, the good and the bad alike suffer. Good and bad alike suffer, because, whatever respect they may have shown to moral laws, in this case they have offended the law of gravity-a law which exacts their lives or their limbs as its penalty. If a man swallow poison, the poison will to its work, whether the act of taking was a conscious or an unconscious act. This knowledge has enabled us to avert danger from our. selves and from those who, like children, would, in their ignorance, swallow the bright and tempting berry merely because it was pleasing to the eye, unconscious of the deadly principles lurking in its core. It is only within a short time that we have learned that men may become dull, listless, unable to command their faculties, because of the poisonous fumes from a fireplace or a stove, or the impalpable powder that floats from the arsenical paper decorating the walls. We know that the green tracery which scatters garlands and vines over our modern walls may be as death-dealing, because of this unseen arsenical presence, as the fumes of carbonic acid gas. We know that a whole district may fall under the terrible scourge of typhoid fever because the water-supply has found some contamination. We know that dinnerguests may be affected by scarlet fever because of ices composed of milk which contained the virus of that disease. ${ }^{*}$ The laws of nature are independent of our fancies. We must conform to them, or suffer the penalty of disobedience and neglect. Ignorance will not prevent disease. A physician's fancies as to the cause of a disease, or the nature of a remedy, will not check it. It is only by knowing as a truth, by patient study and investigation, the exact causes of disease, that we can avoid it. It is only by knowing these causes, the value of the remedies which will affect them, and the conditions of the human frame under which these remedies can have their full influence, that we can effect a cure. How this is to be done, is the subject to which $I$ propose to address myself in the next lecture.

* Buchanan. Mr. Simon's Public Health Report for 1875 .

Among those who passed the recent Cambridge Local Examinations with honours was a lad named Farrar, who is deaf and dumb. He is under sixteen years of age, and has obtained a certificate for classics and mathematics. This is probably the first case of the sort in the United Kingdom.

\section{THE DIAGNOSIS AND TREATMENT OF AUDITORY-} NERVE VERTIGO.*

By W. R. GOWER S, M.D.,

Assistant-Physician to University College Hospital, and to the National Hospital for the Paralysed and Epileptic.

THE form of vertigo which depends on a morbid state of the organ of hearing, and has been variously termed "auditory vertigo", "labyrinthine vertigo", or, from the name of the aural surgeon who first called attention to its more striking characters, "Menière's vertigo" or "Menière's disease", has passed, to some extent, from the province of special aural surgery into that of general medicine. The reason is not far to seek. The symptom vertigo has usually little or no apparent connection with its actual cause. Hence its origin is constantly unsuspected by the sufferer. Moreover, there exists frequently an obtrusive association between this giddiness and certain gastric symptoms, which has con. stantly been and is still frequently misconstrued, and the nature of the disease consequently misconceived by both the patient and his medical adviser. The object of this paper is to illustrate these associations, which so obscure the real relations of the symptom, and to consider the points which are of chief impcrtance in its diagnosis and in its treatment.

It is not necessary here to do more than allude to the evidence on which the pathology of the disease is based. That evidence is of three kinds : first, the frequent association of paroxysmal vertigo with defect or disturbance of the function of the internal ear or auditory nerve secondly, on some scanty pathological facts, which point to the exist. ence in these cases of a morbid state of the semicircular canals ; and, lastly, on the well known experimental evidence of the connection between the function of the semicircular canals and the maintenance of the equilibrium of the body. $t$ The theory of their function is, that they give information of the position of the head to a coordinating centre which presides over the equilibrium of the body, and which experimental physiology has located in the cerebellum. Into the question of the mechanism of the action and disturbance of the semicircular canals it is not necessary here to enter. Although the theory of varying pressure of the endolymph originated by Goltz, and rendered more precise by later writers, appears highly probable, its application to the details of the normal action of the canals is still surrounded by uncertainties which render it difficult to frame a satisfactory detailed explanation of the phenomena of this disease. $\neq$

All that we can conclude is, that their morbid condition causes a morbid action of the centre of equilibration, which may so disturb the equilibrium of the body as to throw the sufferer to the ground, or may merely result in a sense of corresponding movement or of mere instability. It is important, for a clear comprehension of the symptoms of these cases, to remember that the sense of movement and an actual movement, subjective vertigo and actual rotation or falling, are aspects of the same process. It is the motor tendency to turn which is felt as subjective vertigo. This was pointed out long ago by Dr. Reynolds, $\S$ and its significance has been insisted upon from many points of view by Dr. Hughlings Jackson.

Whether any impression from the semicircular canals enters into the total sensation is uncertain, but that the chief part of the feeling of vertigo, that of movement in the individual or in external objects, is the sensation of an "incipient motor process", is evident from the fact

* A portion of this paper was read before the meeting of the British Medical Association at Sheffield, August 1876 .

$\dagger$ An abstract of the facts, with references to the chief sources of information, has been given by Dr. Hughlings Jackson (Medical Times and Gazette, August i7th, 1872 , and London Medical Record, 1874, pp. 238 and 254). The experimental evi-
dence has been fully described and discussed by Dr. Ferrier (Functions of the Brain,

p. 69 , and West Riding A sylum Reports, vol. v).
$\ddagger$ Assuming the theory of Mach, Breuer, and Crum-Brown to be correct, that the $\ddagger$ Assuming the theory of Mach, Breuer, and Crum-Brown to be correct, that the
variation in the pressure of the endolymph in the ampullæ of the several canals, provariation in the pressure of the endolymph in the ampulla of the several canals, pro-
duced by the movements of the head, constitutes the means of their stimulation, duced by the movements of the head, constitutes the means of their stimulation, by plus variations in the pressure of the endolymph in the ampulla. The excitation of by plus variations in the pressure of the endolymph in the ampulla. The excitation of the several ampullary nerves in various movements will therefore be a very complex matter, involving probably the mutual action of several canals, just as the guiding information as to the position of the eyeball, by which the equilibrium centre is also influenced, depends on the degree of contraction of several muscles, oblique and straight. It must be remembered, also, that the free communication between the canals will increase the complexity of their mutual relations.

8 "Generally, in man, the tendency to movement is arrested by an effort of the will per se, or by grasping some object for support. The sensation of its production remains, and is projected onwards into the objects of the material world" (On $V_{\text {er- }}$ tigo, 1854 , p. $4 \mathrm{r}$ )

II "' Giddiness is objectively a motor symptom, and the 'sensation' it is attended by is a state of consciousness accompanying the outgoing current" (Clinical and Physiological Researches on the Nervous System, Preface, p. xxv; see als ) Medical Press and Circular, December and, i874, p. 477 
that the sensation in the slighter attacks is always in harmony with the movement in the severe attacks. If the patient feel as if he were moving, it is towards the side to which he does move in the severe paroxysms. If the sensation be merely one of movement in external objects, it is always from the side towards which the patient falls; i.e., it is the apparent movement of objects (say, to the left) which would result from an actual movement of the head or eyes to the right. 'In a case to be presently narrated, this was very marked. The practical significance of this is, that the character of the vertigo is an important aid in the diagnosis of its cause, and we are able to determine its character as accuracely from such a subjective sensation as if we saw the patient reel. Hence the importance of ascertaining in every case of giddiness the precise character of the patient's sensation.

The derangement of hearing with which this form of vertigo is associated may be of two kinds. Sometimes there is evidence of undue excitation of the auditory nerve, noises in the ears, permanent or only at the moment of the paroxysm. More frequently, indeed almost universally, there is evidence of defective sensitiveness. This-defect may be conspicuous or obscure; may range from a considerable degree of deafness to a slight defect in audition, which it requires much care to ascertain. The knowledge that the defect may be limited to the perception of sound conducted through the bones of the skull is an important addition to our means of diagnosis. The fact was pointed out by the late Mr. Hinton* and by Mr. Dalby, $†$ and cases in which it was noted have been published by Dr. Ferrier, Dr. Duffin, and others. This loss of what may conveniently be termed perosseal audition is regarded as evidence of an affection of the labyrinth or of the auditory nerve, may be absolute; a tuning-fork vibrating in contact with any part of the head or with the teeth may be unheard in the affected ear, while it is heard readily, if held opposite the meatus ; or the loss may be partial, and in that case it may be detected by the methud, commonly employed by aural surgeons, of closing the ears while the tuningfork is sounding in contact with the vertex or some other part of the skull. Closure of the meatus; if the latter be unobstructed, increases the intensity of the sound, if the perception of sounds conducted through the skuld be unimpaired, renders it weaker or inaudible, if there be impairment. But the method is one that requires much care and repeated examination to detect a slight defect in an unintelligent patient. It is important, however, to examine these cases, not only with a tuning fork, but with a watch. There may exist marked impairment of perosseal audition to the watch, while the tuning-fork is well heard. $f$ I know a gentleman who has been subject for several years to slight attacks of vertigo. The motion is uniform, a tendency to fall to the left. His hearing is acute; it was thought to be perfect. A tuning-fork in contact with the head is heard perfectly well with ears open or closed. A watch placed opposite the ear is heard perfectly well ; but the loudest ticking watch pressed against any part of the head is not heard in the least. The condition, however, is a variable one. At times, a watch in contact with the head is heard well ; more frequently it is not heard at all. The liability to attacks of vertigo seems to correspond to the periods of imperfect audition. Whenever he has been tested after an attack, the power of hearing the watch has been always absent.

Even a siighter degree of impairment of the power of hearing a watch in contact with the skull may be of significance, as is shown by the:following case lately under my care, in which the tuning-fork failed to reveal any abnormality in the function of hearing.

P. D., aged 56, admitted into University College Hospital on August I 5 th, 1876 , had suffered during the last five years from startings on going to sleep, which lately occurred every night. Four months previously, he had a blow on the head and on the bridge of his nose from the fall of plaster. In June (two months before admission), he noticed a considerable degree of deafness of the left ear, which continued for a fortnight and then passed away. During the six days before admission, a confused noise in the left ear was noticed. Twice during the preceding month, he had an attack of giddiness of short duration, of which no definite history could be obtained. The day before his admission, while walking, he suddenly became giddy, as if he were turning round, stag. gered towards the right side, and fell, as he says, in trying to recover

\footnotetext{
* On Labyrinthine Vertigo (Guy's Hospital Reports, 1873).

+ Lectures on Diseases of the Ear, 1873 .

'The method of testing perosseal audition with the watch which $I$ have found most useful, is to hold the watch first close to, and then in firm contact with, the zygoma, the parietal eminence, and the mastoid process-the direct passage of the vibrations to the meatus being prevented by placing the hand as a screen between the watch and the ear in the two former positions, and in the latter by drawing forward the pinna. The effect of contact in the normal state is very marked. The watch not in contact is scarcely heard or inaudible, while in contact it is loud. This method of testing is somewhat coarse, but very convenient. The effect of closure of the meatus on the sound of a watch is slighter than in the sound of a tuning-fork; and although in health it is distinct, it is not sufficient to render this test a convenient one.
}

himself.' A slight sense of movement on his part and in surrounding objects continued until his admission. He then complained much of a "confusion of sounds", which seemed to be in his head, and of headache in the occipital region and behind the ears. His hearing at first seemed to be natural. A watch was heard a good and equal distance from each ear. A tuning-fork was heard at eight feet, and was well heard when sounding in contact with the head in various positions, and in each ear it was increased in loudness by closing the meatus. A watch was heard in contact with the head and on the right side; closing the meatus increased its loudness. On the left side, however, closing the ear rendered the sound less loud; the watch had a double beat, and closure of the ear rendered one. set of beats entirely inaudible. This result was obtained in every examination. (The patient was a very in. telligent man, and his answers seemed reliable.). A few days after his admission, he had another severe attack of giddiness, with great "confusion of sounds in his left ear". His rest at night was much disturbed, and he turned about in his sleep until his head came to rest at the foot of the bed. Sometimes he lay curled round, with his head hanging over the edge of the bed. If he were awakened and put straight, on going to sleep again he soon returned to the former position. Five days later, he had another attack of vertigo, which continued in a slighter form for some hours, and some peculiar clonic spasm was noticed in the right arm and leg. He appeared able to control it when his attention was directed to it ; but, when his attention was otherwise engaged, the spasmodic movement went on, the right hand being continually jerked up and down, so that the hand kept striking the epigastrium. At this time, he said he felt as if he were falling through the bed or swimming about the ward, so that an effort was necessary before he could realise that he was in bed. During the height of the attack, he complained of a continuous whirling or humming noise in both ears. A dose of chloral and bromide of potassium quieted him, and, on bromide of potassium and iron, he had no other severe attack while in the hospital

In this case, the change in the hearing was so slight, that hesitation might well be felt in assigning any significance to it, were it not that the previous deafness on that side, and the noises in the ears which accom. panied the severer attacks of vertigo give weight to the auditory defect, slight as it is. The occurrence of quasi-convulsive movements is a point of much interest, to which I will return. The remarkable postures assumed by the patient in his sleep are curious, and may perhaps be connected with the altered action of the organs or centres of equi. libration. May the sudden startings on going to sleep be associated with the same condition? It is a well known fact that they are often related to a distinct dream of defective equilibrium.

But slight impairment of the power of hearing a watch or a tuning fork, when vibrating in contact with the head, is not uncommon, even in persons who do not habitually suffer from vertigo. This is not surprising, since it is almost certain that the apparatus for the recognition of sound and of the position of the body are not the same, although they may be adjacent and even connected and supplied by portions of the same nerve. Even supposing, with Dr. Ferrier, that the semicircular canals are the channels by which the vibrations conducted through the bone reach the cochlea, we do not know that all disturbances of the semicircular canals cause vertigo. But I think some facts to be adduced render it probable that derangement of these canals may bring the centre of equilibration into an unstable condition, in which it is easily excited to sudden perverted action (paroxysmal vertigo) by some abnormal impression on the other nerves with which it is connected. It seems, therefore, that a morbid state of the semicircular canals may predispose to yertigo, as well as excite it. I shall return to this in speaking of the diagnosis of auditory and gastric vertigo, and shall mention some instances in which the attacks of vertigo appeared clearly predisposed to by aural, and excited by gastric, disturbance. A probable example of such a relation was afforded by a member of our profession, who, on two occasions near together, was seized with intense vertigo, brief but definite in character, the sensation being on each occasion that of a similar rotation. The attacks occurred after and were attributed to the inhalation of tobacco-smoke into the lungs. On subsequent examination of the sense of hearing, it was found that, while both tuning-fork and watch were heard when in contact with the head, closure of the left meatus rendered the sound considerably louder, while closure of the right meatus made little difference in the intensity of the sound. He had suffered from some ear-trouble in the right ear, after scarlet fever in early life; the ear was deaf for a time, but gradually, in the course of years, regained normal power. It is possible that, in this case, some permanent defect in the function of the semicircular canals, which being slight, or being compensated or allowed for in the sensori-motor adjustments, did not cause vertigo, may yet have induced a state of defective stability in the centre for equilibrium, which the 
strong impression on the pulmonary pneumogastric nerve sufficed to overturn. The relation of the pneumogastric to the function of equilibration is well known. Dr. Hughlings Jackson has pointed out that the connection of its nucleus with that of the auditory nerve in the medulla, may explain the frequent occurrence of vomiting in the paroxysms of Menierre's disease; and Dr. Ferrier has suggested that there is probably a still higher association between the two nerves in the equilibrial centre.

The vomiting which accompanies the disturbance of equilibrium, and which is to be attributed to the association with the function of the pneumogastric, is the source, as already stated, of much of the misconception as to the nature of these cases. A few years ago, had the question been asked, What are the commonest causes of paroxysmal vertigo ? the invariable answer would have been "A disordered stomach or a diseased brain". It is probable that, even now, the exceptions to such an answer would be comparatively rare. The answer, at any rate, indicates the conditions from which the diagnosis of auditory-nerve vertigo has to be made; for gastric and cerebral disturbances are probably, next to labyrinthine affections, the most common causes of giddiness, and the paroxysms of Meniere's vertigo are constantly ascribed to one or the other of those conditions.

The gastric associations are especially liable to mislead. The occurrence of vomiting, in the absence of other obtrusive cause of the vertigo, is held as proof of a causal derangement of the stomach. Sometimes, an attack of vertigo may not cause vomiting; it may merely disturb the gastric functions, just as the motion of a ship does with some persons in whom it does not cause seasickness. The dyspepsia which results is regarded as a sufficient cause for the giddiness. But, further, in persons the subjects of auditory vertigo, whose equilibrium nerves and centre are deranged, a primary gastric disturbance seems sometimes to excite a paroxysm of the special vertigo to which they are liable, and the gastric disturbance which thus causes the attack is naturally regarded as its only antecedent.

Anillustration of this is afforded by the case of a gentleman, whosuffers irom frequent attacks of intense dyspepsia and vertigo ; the former appearing to him to precede and cause the latter. The vertigo is sudden and violent ; he has fallen with it, and would fall always if he were not careful. He cannot say to what side he falls; but on one occasion must have fallen to the right, as he grazed the right side of his cheek. $\mathrm{He}$ is almost completely deaf on the right side; a watch, in contact with the skull on that side, is quite inaudible. The hearing on the other side seems unimpaired. The deafness came on gradually about twelve years ago. He has been subject from boyhood to attacks of violent dyspepsia, with vomiting and prostration. In his youth they were as severe as they are now; but he never suffered from vertigo until the onset of his deafness. Since that time, the dyspepsia and vertigo have gone on together. The paroxysms of vertigo in this case appear distinctly to be excited on the attacks of gastric disturbance. They are produced by errors in diet, and the long liability to such dys. pepsia indicates that it is not to be regarded as secondary to the aural affection. But the sequence of the symptom, the coincidence of the liability to vertigo and of the ear-disease, indicate clearly that the giddiness is ultimately due to the influence of the latter, although it is excited by the stomach-derangement.

$$
\text { [To be continued.] }
$$

\section{A NEW THEORY OF THE ORIGIN OF TYPHOID FEVER.}

By W. STEWART, L.R.C.P. \& L.R.C.S.Ed., Honorary Surgeon, Beckett Hospital, Barnsley.

AT the present time, when the professional mind is carefully feeling after the origin of typhoid fever, I presume any probable theory that may be advanced to explain many of the facts surrounding this important question will in some degree be of use to, and therefore be welcomed by, those who make the subject their special study. It is with this hope that I venture to submit the following facts upon which I build my theory.

In attempting to discover the cause of this fever, from the fact of its universal prevalence in hot and cold climates, in town and country, in the houses of the rich and in the hovels of the poor, it is necessary to fix the blame upon a factor that is equally ubiquitous; and I would further add that, during the period in which my thoughts have been directed to the subject, the accumulation of different evidence bearing upon it has only tended, in my opinion, to increase the probability of the theory.

Three years ago, I attended seven or eight cases of this disease in a row of six cottages situated on the crest of a hill about two miles from this town. After careful inquiry, I arrived at the conclusion that the cause of this outbreak was to be attributed to the fact that the slaughter-house of a butcher was situated at the end of the row, into the common sewer of which the blood from his operations was allowed to flow, there to remain and putrefy. The waste-pipes from the sinks were directly connected with this drain without the intervention of any kind of trap, and the smell therefrom was often horrible. Here, the putrefaction of a highly albuminous liquid, blood, in the drain, and a direct communication between it and the interior of the cottages, seemed to give rise to the fever.

The following case, related by Dr. Domenichetti, medical officer of health, Louth Sanitary District (Public Health for July 1875), and quoted by Dr. Cornelius Fox (BRITISH MEDICAL Journal, vol. ii, 1875, p. 376), appears to have had a similar origin ; viz., the introduction of the products of decomposing blood to the system; in this case, by the water-supply. "A man aged 57 was seized with marked symptoms of typhoid fever, which terminated fatally in about a week, and, upon investigation, it appeared that the drinking water was at fault, though not suspected at the time, as it was derived from a blowwell, which, in these districts, is a term applied to water from the calcareous rock."... "Upon examining the premises, I ascertained there was a stable-grating within a few yards of the well, and I requested the owner to have the drain opened. Upon this being done, the tin pipe through which the water was conducted from the underlying rock was found to be corroded through, and at its base there was an accumulation of refuse from pig-slaughtering, etc., quite sufficient to account for the contamination of the water. I should observe that no other case of typhoid fever had occurred near the spot for some time."

In the experiments instituted by M. V. Feltz, and communicated to the Académie des Sciences, upon the effect produced upon dogs by the injection of putrid blood, and alluded to in a contemporary (Lancet, vol. $\mathrm{ii}, \mathrm{I} 875$, p. 460 ), the symptoms produced were very analogous to those we see in typhoid fever.

"Putrid blood which had stood for several months was dried and desiccated in the air-bath and mixed with a certain quantity of distilled water, and injected into the crural vein of three powerful dogs. The animals immediately exhibited marked depression. After a period of incubation of from four to five days, febrile symptoms set in, accompanied by vomiting, loss of appetite, elevation of temperature, bilious and bloody diarrhoea, and biliary urine", and these symptoms were produced even when all trace of bacterial life had disappeared from the blood injected.

Pondering over the origin of the typhoid cases first mentioned in this paper, my thoughts were directed to the analogies existing between the symptoms present in typhoid fever and those which characterise certain cases of puerperal fever which appear to arise from the introduction of the product of decomposing blood to the system by a channel very much the same as in the case of the experiments on the dogs. In the cases to which I allude, diarrhoea is often one of the first appreciable symptoms, accompanied by high fever, the peculiar typhoid tongue, and, later on, hurried respiration, showing that marked tendency to affection of the lungs which is common to both.

I remember a case of this form of puerperal affection that occurred in a primipara after delivery by the forceps. Two days after delivery, she had a rigor accompanied by severe pain in the abdomen; she recovered after an illness of four weeks, during which time she suffered from symptoms remarkably like an ordinary typhoid attack in all the more important particulars. Unfortunately, as a general rule, cases attacked like this die in the course of a week or ten days, and the further clinical study of them is thus effectually prevented; but so much have some of them resembled ordinary typhoid fever cases of a very severe type that, if it were possible to have overlooked the puerperal condition, they might have been set down as cases of that fever. Of course, the puerperal cases are accompanied by local affections of the organs through which the poison travels before it finally reaches the general body of circulating blood, and this fact seems for a considerable time to have obscured the true nature of these cases by diverting attention from the ultimate general effects upon the system and fixing it too exclusively upon the local expressions of the infection.

The different channels by which the poison is admitted to the blood -in the puerperal case through the lymphatics or veins of the uterus, in the typhoid case through the stomach-would to my mind go far to account for any apparent variations in the symptoms. By the first method, the decomposing albuminous matter is admitted more directly, less changed by any chemical process, and in a more recently putrefied, and therefore in a more active condition, to the blood, and thus produces, as we might have expected, much severer effects than when it 